\title{
"Mini-Flow-Through" Deep Inferior Epigastric Perforator Flap for Breast Reconstruction with Preservation of Both Internal Mammary and Deep Inferior Epigastric Vessels
}

\author{
Toshihiko Satake ${ }^{1}$, Jun Sugawara ${ }^{2}$, Kazunori Yasumura ${ }^{1}$, Taro Mikami ${ }^{2}$, Shinji Kobayashi ${ }^{3}$, \\ Jiro Maegawa ${ }^{2}$ \\ ${ }^{1}$ Department of Plastic and Reconstructive Surgery, Yokohama City University Medical Center, Yokohama; ${ }^{2}$ Department of Plastic and \\ Reconstructive Surgery, Yokohama City University Hospital, Yokohama; ${ }^{3}$ Department of Plastic and Reconstructive Surgery, Kanagawa \\ Children's Medical Center, Yokohama, Japan
}

This procedure was developed for preservation of the rectus muscle components and deep inferior epigastric vessel after deep inferior epigastric perforator (DIEP) flap harvesting. A 53-year-old woman with granuloma caused by silicone injection underwent bilateral nipplesparing mastectomies and immediate reconstruction with "mini-flow-through" DIEP flaps. The flaps were dissected based on the single largest perforator with a short segment of the lateral branch of the deep inferior epigastric vessel that was transected as a free flap for breast reconstruction. The short segments of the donor deep inferior epigastric vessel branch are primarily end-to-end anastomosed to each other. A short T-shaped pedicle mini-flow-through DIEP flap is interposed in the incised recipient's internal mammary vessels with two arterial and four concomitant venous anastomoses. Although it requires multiple vascular anastomoses and a short pedicle for the flap setting, the mini-flow-through DIEP flap provides a large pedicle caliber, enabling safer microsurgical anastomosis and well-vascularized tissue for creating a natural breast without consuming time or compromising the rectus muscle components and vascular flow of both the deep inferior epigastric and internal mammary vessels.

Keywords Mammaplasty / Perforator flap / Microsurgery / Granuloma / Subcutaneous mastectomy
Correspondence: Toshihiko Satake Department of Plastic and Reconstructive Surgery, Yokohama City University Medical Center, 4-57 Urafune-cho, Minami-ku, Yokohama, Kanagawa 232-0024, Japan

Tel: +81-45-261-5656

Fax: +81-45-253-5375

E-mail: toshi@yokohama-cu.ac.jp

No potential conflict of interest relevant to this article was reported.

Received: 15 Jun $2015 \bullet$ Revised: 21 Aug $2015 \bullet$ Accepted: 14 Sep 2015

pISSN: 2234-6163 • elSSN: 2234-6171 • http://dx.doi.org/10.5999/aps.2015.42.6.783 • Arch Plast Surg 2015;42:783-787

\section{INTRODUCTION}

The deep inferior epigastric artery perforator (DIEP) flap is frequently used in autologous breast reconstruction procedures and has recently been used as a substitute for the free transverse rec- tus abdominis musculocutaneous (TRAM) flap [1] because it provides thick, well-vascularized skin and adipose tissue with a long and large caliber pedicle. Although it preserves the rectus muscle component during flap elevation, there exists a risk of abdominal complications, such as abdominal bulging or hernia [2]. 
To reduce donor-site morbidity after a conventional long pedicle DIEP flap, we introduce some modifications in flap harvesting, pedicle transection, microsurgical anastomosis, and flap setting. This technique finally leads to the preservation of the vascular flow of both the internal mammary vessel and deep inferior epigastric vessel after a DIEP flap breast reconstruction.

\section{IDEA}

With the goal of preserving the rectus muscle and deep inferior epigastric vessel during DIEP flap harvesting, we dissected the flap based on its single largest perforator with a short segment of the medial or lateral branch of the deep inferior epigastric vessel and then transected it as a free flap for breast reconstruction.
The proximal and distal stumps of the donor deep inferior epigastric vessel branch were primarily end-to-end anastomosed to each other. The short T-shaped pedicle "mini-flow-through" DIEP flap is interposed in the incised recipient internal mammary vessels with two arterial and four concomitant venous anastomoses (Figs. 1, 2).

Thereafter, the donor-site incision in the anterior rectus sheath is closed without any tension, and the transferred DIEP flap is tailored to create a natural breast size and shape.

A 53-year-old female with a lower abdominal midline scar due to a previous cesarean section complained of bilateral breast lumps for 5 years and recent pain. She had received liquid silicone injections for bilateral breast augmentation approximately 30 years earlier [3]. On examination in our outpatient clinic, the

\section{Fig. 1. Schematic of mini-flow-through DIEP flap breast reconstruction}

Prepare the recipient site (IMV), then elevate a mini-flow-through deep inferior epigastric artery perforator flap (DIEP) flap, with a short segment of T-shaped pedicles, at the level of the perforator from a lateral or medial branch of the deep inferior epigastric vessel. After harvesting the flap, repair the transected deep inferior epigastric vessel primarily with end-to-end anastomosis. Then, a short T-portion mini-flow-through DIEP flap pedicle is anastomosed end-to-end to both the proximal and distal stumps of the IMV. (A) Flap harvesting. (B) Microsurgical anastomosis.

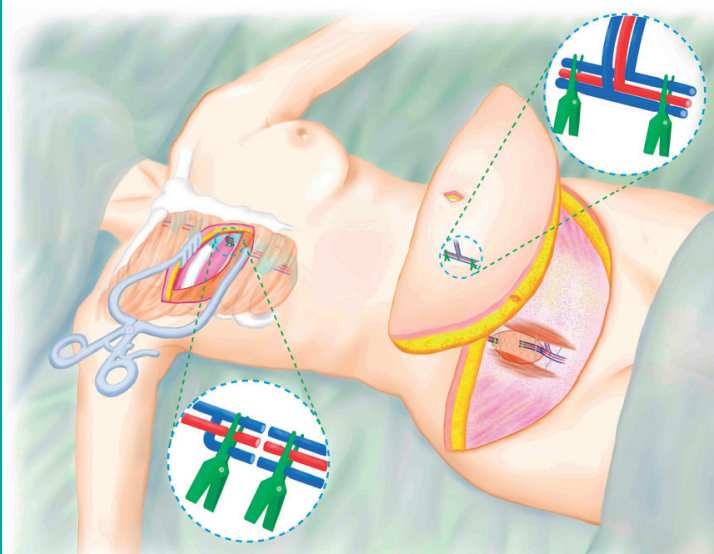

(A)

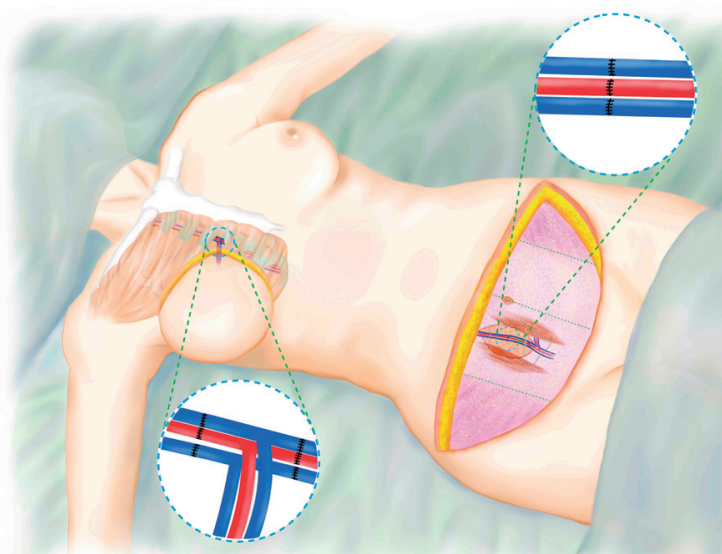

Fig. 2. Intraoperative view of mini-flow-through DIEP flap

After harvesting bilateral deep inferior epigastric artery perforator flap (DIEP) flaps with short T-shaped pedicle, the transected deep inferior epigastric vessel (DIEV) branch was repaired with a primary end-to-end anastomosis. Then, these short T-shaped pedicles were anastomosed end-toend to both the proximal and distal stumps of the incised recipient internal mammary vessels (IMVs) in the fourth intercostal space. (A) A miniflow-through short pedicle placed on a small square of blue paper. (B) Repaired DIEV with end-to-end anastomosis (white arrow). (C) Two endto-end anastomosis points between the incised IMV and a short T-shaped pedicle (white arrows). (Reproduced from Satake, et al. J Jpn Soc Plast Reconstr Surg, with permission Japan Society of Plastic and Reconstructive Surgery [3]).
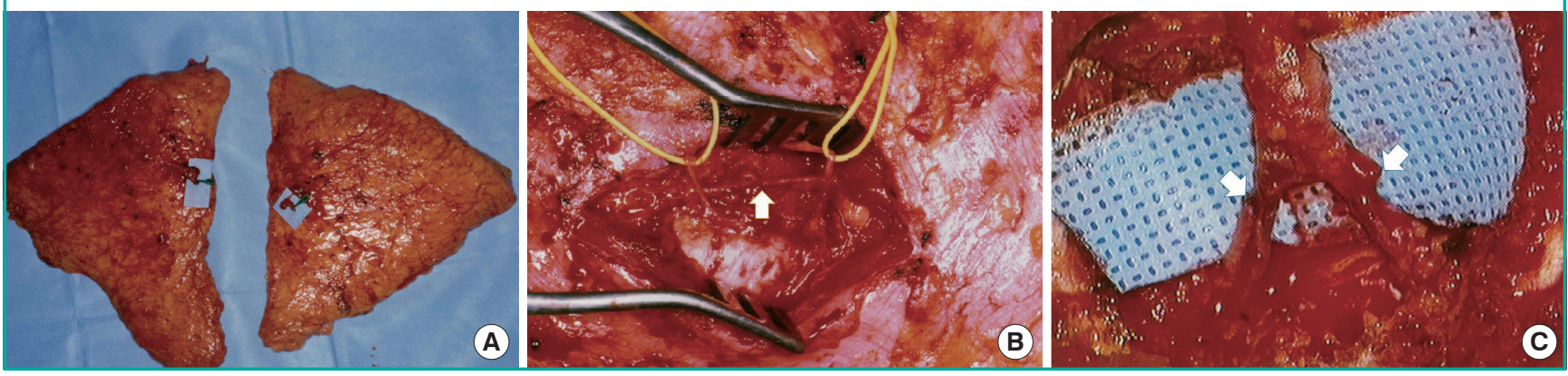
breasts were lumpy, deformed, and occasionally tender on palpation (Fig. 3A). No inflammatory lesion over the breast skin or palpable axillary lymph nodes were noted. Mammography and magnetic resonance imaging showed no evidence of breast cancer. A diagnosis of bilateral silicon granulomas of the breast was made. The patient was offered the choice of surgical treatment and consented to undergo bilateral mastectomies and breast reconstruction.

The DIEP flaps were designed on the patient's lower abdomen (Fig. 3B) in a $11 \mathrm{~cm} \times 40 \mathrm{~cm}$ area. Under general anesthesia, a skin incision was made first through the bilateral inframammary line that connected at the midsternum. Such an inframammary approach made it easy to perform bilateral mastectomies, expose the recipient internal mammary vessels, and perform flap inset. Both the silicon granulomas in the mammary glands were resected, sparing the normal subcutaneous tissue, breast skin envelope, and the nipple-areola complex. After the skin paddle for the bilateral DIEP flaps was incised down to the abdominal deep fascia, the largest lateral row perforator on each side was detected and preserved from among the several perforators present bilaterally. Through a small incisional window in the anterior rectus sheath that measured approximately $5 \mathrm{~cm}$, the perforator that connected the intercostal vessels, nerves, and lateral branch of the deep inferior epigastric vessels was separated by deep dissection in the posterior sheath. A short segment of the lateral branch of the deep inferior epigastric vessel, measuring
$1.5 \mathrm{~cm}$, including a perforator, was transected from the donor deep inferior epigastric vessel without any further dissection of the external iliac vessel (Fig. 2A). The flap pedicles were approximately $2.5 \mathrm{~cm}$ each. Further, the donor proximal and distal stumps of the lateral branch were primarily repaired with a tension-free end-to-end anastomosis (Fig. 2B). At the base of the flap pedicle, the adipose tissue was temporarily fixed to the split pectoralis major muscle. Subsequently, the other end of the flap was flipped over so that the surgical field was clear for easier anastomoses. After the short T-shaped pedicle of the mini-flowthrough DIEP flap was interposed between the incised internal mammary vessels in the fourth intercostal space with both antegrade and retrograde anastomosis, all stumps from one artery and two concomitant veins were anastomosed for flap revascularization (Fig. 2C). The flaps were revascularized via "flowthrough": anastomosed in the order of the medial internal mammary vein, internal mammary artery, and lateral internal mammary vein, using the back wall technique. Both the de-epithelialized DIEP flaps were then arranged to reconstruct the breast. During revascularization through microsurgical anastomosis, the external caliber, ranging from $1.0 \mathrm{~mm}$ to $1.5 \mathrm{~mm}$, of the deep inferior epigastric and internal mammary vessels was assessed to be sufficiently large to permit safer anastomosis. The total operative time was 12 hours by a single-team approach (two surgeons, each working on one side of the bilateral procedure).

Histopathology of the surgical specimens revealed nonmalig-

Fig. 3. Preoperative and postoperative appearances of the patient

A 53-year-old woman with a lower abdominal midline scar due to a previous cesarean section had bilateral silicone granulomas of the breast. Her breasts were reconstructed with bilateral mini-flow-through deep inferior epigastric artery perforator flap (DIEP) flaps after nipple-sparing mastectomies. (A) Preoperative view. (B) Preoperative designs of bilateral nipple-sparing mastectomies and bilateral DIEP flaps. (C) Postoperative view. (Reproduced from Satake, et al. J Jpn Soc Plast Reconstr Surg 2003;23:28-37, with permission Japan Society of Plastic and Reconstructive Surgery [3]).
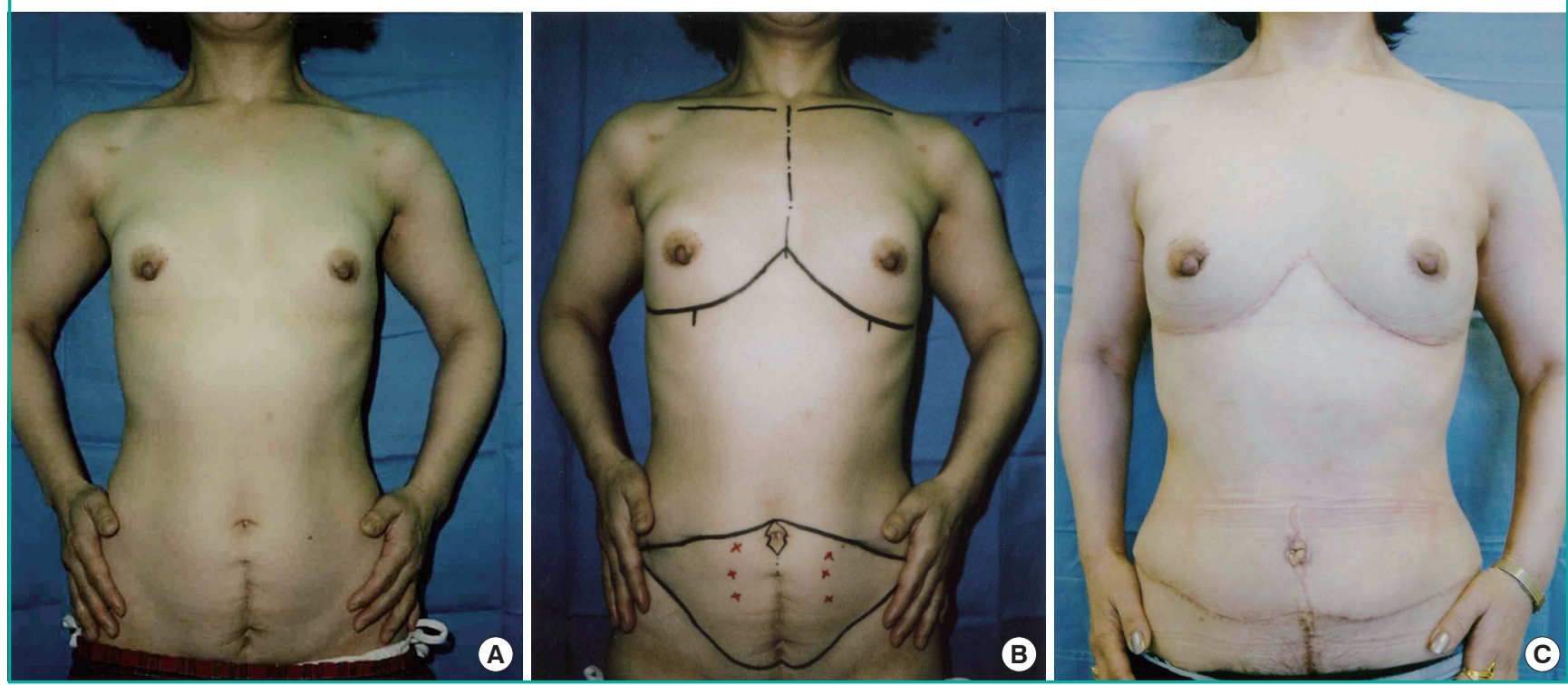
nant atypical cellular changes. The postoperative course was uneventful, without complications at either the donor or recipient sites. The patient was satisfied with the results of breast reconstruction (Fig. 3C).

\section{DISSCUSSION}

Since the DIEP flap was developed in 1989 [4], some modifications in pedicle shortening have been made to reduce donor-site morbidities and the long duration of surgery. Although Koshima et al. [5] harvested a DIEP flap (paraumbilical perforator flap) with only a true perforator pedicle, it required supermicrosurgical skill for revascularization. Schoeller et al. [6] introduced a modified paraumbilical perforator flap with an approximately 7- $\mathrm{cm}$ pedicle length that seemed to be adequate for simple microsurgery. Similarly, Colohan et al. [7] reported longer pedicle DIEP flaps, ranging from 8.1 (ultrashort) to $10.4 \mathrm{~cm}$ (short) in pedicle length. In contrast to Koshima's modified methods [5], the mini-flow-through DIEP flap, despite requiring an incision in the anterior rectus sheath, is minimally invasive and less time consuming. Moreover, the flap offers larger caliber stumps of arterial and venous pedicles, enhancing the feasibility of safer microsurgical anastomosis.

The mini-flow-through DIEP flap has a shorter pedicle length in contrast to that reported in the latter two procedures $[6,7]$ that makes it difficult to perform flap setting similar to that used for a superficial inferior epigastric artery flap. However, it enables preservation of the normal vascular flow of the deep inferior epigastric vessels with primary anastomosis because the gap in the deep inferior epigastric vessel is only $1.5 \mathrm{~cm}$. A short segment of the nutrient vessel, including a T-shaped perforator pedicle flap, was first introduced in a distally based radial artery perforator free flap $[8,9]$ to preserve the integrity of the radial artery. Bauer et al. [8] anastomosed this short T-shaped pedicle to the recipient vessel using a mini-flow-through technique, and we referred to their method in our clinical case.

The mini-flow-through anastomosis to the internal mammary vessel resembles the end-to side anastomosis to the internal mammary vessel [10] or the end-to-end anastomosis to the internal mammary intercostal perforators [11]. These procedures preserve the vascular flow of the internal mammary vessel while allowing shorter pedicles than with the thoracodorsal vessel and medial placement of the flap while avoiding axillary scarring [12]. Moreover, the preserved internal mammary vessel could prove to be a source vessel for a future cardiac bypass. In 2010, there were 395,000 coronary artery bypass graft procedures conduced in the United States [13]; therefore, plastic surgeons should consider the patient risk of coronary artery disease and the potential use of the internal mammary vessel when planning microsurgical breast reconstruction.

End-to-end flow-through anastomosis has a number of advantages over end-to-side anastomosis. Koshima et al. [14] reported that a flow-through short pedicle-free flap has a high success rate, which is attributable to duplicated vascular anastomosis with double arterial inflows and two or more vessels for venous drains including two retrograde drains. We believe that this makes flap circulation secure and stable. During DIEP flap harvesting, many branches from the deep inferior epigastric vessel are encountered. After arising from the external iliac vessel, the deep inferior epigastric vessel initially sends a recurrent nutrient branch to the rectus muscle medially. The major portion of the deep inferior epigastric vessel divides into medial and lateral branches [15] that then send several large perforators below the level of the umbilicus. The DIEP flap can be used as a flowthrough free flap at these branching portions. Among these large perforators, both limbs of the deep inferior epigastric vessel branch may be appropriate candidates to be used for connecting the largest perforator in this procedure.

The conventional long-pedicle DIEP flap requires more time for dissection during flap harvesting, and a large incisional window in the anterior rectus sheath, while involving tedious dissection, possible damage to the rectus muscle, and loss of the main nutrient vessel. Our modified breast reconstruction procedure is less invasive after harvesting of bilateral DIEP flaps. Under such circumstances, the mini-flow-through DIEP flap may be an alternative in autologous breast reconstruction procedures.

Although it requires multiple vascular anastomoses and a short pedicle for flap setting, the mini flow-through DIEP flap provides a large pedicle caliber, enabling safer microsurgical anastomosis. It also provides well-vascularized tissue for creating a natural looking breast, minimizes dissection of the rectus muscle component, and preserves vascular flow of both the deep inferior epigastric and internal mammary vessels. Altogether, it significantly shortens the operative time for flap harvesting.

\section{REFERENCES}

1. Allen RJ. Perforator flaps in breast reconstruction. In: Spear SL, editor. Surgery of the breast. Philadelphia: LippincottRaven; 1998. p.605-25.

2. Mennie JC, Mohanna PN, O’Donoghue JM, et al. Donorsite hernia repair in abdominal flap breast reconstruction: a population-based cohort study of 7929 patients. Plast Reconstr Surg 2015;136:1-9.

3. Satake T, Torikai K, Takahashi T, et al. Experience using the deep inferior epigastric perforator (DIEP) flap for breast re- 
construction. J Jpn Soc Plastic Reconstr Surg 2003;23:28 37.

4. Koshima I, Soeda S. Inferior epigastric artery skin flaps without rectus abdominis muscle. Br J Plast Surg 1989;42:645-8.

5. Koshima I, Inagawa K, Yamamoto M, et al. New microsurgical breast reconstruction using free paraumbilical perforator adiposal flaps. Plast Reconstr Surg 2000;106:61-5.

6. Schoeller T, Bauer T, Gurunluoglu R, et al. Modified free paraumbilical perforator flap: the next logical step in breast reconstruction. Plast Reconstr Surg 2003;111:1093-8.

7. Colohan S, Maia M, Langevin CJ, et al. The short- and ultrashort-pedicle deep inferior epigastric artery perforator flap in breast reconstruction. Plast Reconstr Surg 2012;129:331-40.

8. Bauer TR, Schoeller T, Wechselberger G, et al. The radial artery perforator free flap. Plast Reconstr Surg 1999;104:885.

9. Safak T, Akyurek M. Free transfer of the radial forearm flap with preservation of the radial artery. Ann Plast Surg 2000; 45:97-9.

10. Apostolides JG, Magarakis M, Rosson GD. Preserving the internal mammary artery: end-to-side microvascular arterial anastomosis for DIEP and SIEA flap breast reconstruction.
Plast Reconstr Surg 2011;128:225e-232e.

11. Follmar KE, Prucz RB, Manahan MA, et al. Internal mammary intercostal perforators instead of the true internal mammary vessels as the recipient vessels for breast reconstruction. Plast Reconstr Surg 2011;127:34-40.

12. Moran SL, Nava G, Behnam AB, et al. An outcome analysis comparing the thoracodorsal and internal mammary vessels as recipient sites for microvascular breast reconstruction: a prospective study of 100 patients. Plast Reconstr Surg 2003; 111:1876-82.

13. Mozaffarian D, Benjamin EJ, Go AS, et al. Heart disease and stroke statistics--2015 update: a report from the American Heart Association. Circulation 2015;131:e29-322.

14. Koshima I, Fujitsu M, Ushio S, et al. Flow-through anterior thigh flaps with a short pedicle for reconstruction of lower leg and foot defects. Plast Reconstr Surg 2005;115:155-62.

15. Rozen WM, Palmer KP, Suami H, et al. The DIEA branching pattern and its relationship to perforators: the importance of preoperative computed tomographic angiography for DIEA perforator flaps. Plast Reconstr Surg 2008;121:367-73. 